\title{
Do modern family planning methods impact women's quality of life? Jordanian women's perspective
}

\author{
Mohammad S. Alyahya ${ }^{1 *}$, Heba H. Hijazi ${ }^{1}$, Hussam A. Alshraideh², Nihaya A. Al-sheyab³ ${ }^{3}$ Dana Alomari ${ }^{1}$, \\ Sara Malkawi ${ }^{1}$, Sarah Qassas ${ }^{1}$, Samah Darabseh ${ }^{1}$ and Yousef S. Khader ${ }^{4}$
}

\begin{abstract}
Background: Although Jordan has made progress in meeting Family Planning (FP) needs in last decades, recently the use of FP methods has declined significantly. Women's personal experiences, knowledge, and perceptions of how a FP method might impact their quality of life (QoL) can influence FP decisions. However, a lack of comprehensive understanding of the impact of modern FP methods on women's QoL continues to exist among Jordanian couples. Therefore, this study aimed to investigate the relationship between the use of common modern FP methods and QoL among Jordanian women.

Methods: Using the WHOQOL-BREF questionnaire along with other questions, non-pregnant women of reproductive age were interviewed at their homes through face-to-face structured interviews. Women who visited the obstetrics and gynecology clinic of King Abdullah University Hospital for contraceptive advice and follow-up consultations were also included.

Results: A total of 548 women aged between 18 and 49 participated in the study. Based on the WHOQOL-BREF scale, the overall mean (SD) scores of the four domains were found to be average. Our findings show that women who used Intra Uterine Devices (IUDs) and women whose husbands used condoms had better QoL in the four domains (physical health, psychological health, social relationships, and environment) than those who used Oral Contraceptives (OCs). Women who used implant and injectable hormonal contraceptives had better QoL in terms of the physical health and social relationships domains. In contrast, women who had undergone permanent sterilization had lower QoL scores in all of the four domains. Further analysis revealed that women who had undergone tubal sterilization were less satisfied overall and more likely to experience side effects than women who used OCs.

Conclusion: The choice to use contraceptives and decide freely whether and when to have children is regarded as a fundamental reproductive health right and is strongly linked to women's health and QoL. Women who use OCs and women who have undergone permanent sterilization are likely to have lower QoL than women who use IUDs or implant and injectable hormones and those whose husbands use condoms.
\end{abstract}

Keywords: Quality of life, QoL, Contraceptives, Family planning methods, WHOQOL-BREF, Jordan

\footnotetext{
* Correspondence: Msalyahya@just.edu.jo

${ }^{1}$ Department of Health Management and Policy, Faculty of Medicine, Jordan

University of Science and Technology, P.O.Box 3030, Irbid 22110, Jordan

Full list of author information is available at the end of the article
}

(c) The Author(s). 2019 Open Access This article is distributed under the terms of the Creative Commons Attribution 4.0 International License (http://creativecommons.org/licenses/by/4.0/), which permits unrestricted use, distribution, and reproduction in any medium, provided you give appropriate credit to the original author(s) and the source, provide a link to the Creative Commons license, and indicate if changes were made. The Creative Commons Public Domain Dedication waiver (http://creativecommons.org/publicdomain/zero/1.0/) applies to the data made available in this article, unless otherwise stated. 


\section{Background}

Modern family planning (FP) methods have become more broadly accepted and used as a replacement for traditional methods and as one of the interventions to most empower women. Women empowerment, as an important goal for achieving national development goals, needs to be strengthened and sustained to ensure that women exercise their responsibilities and enjoy their human rights [1-3]. Women status and empowerment have been found to be associated with longer birth interval, lower rate of unintended pregnancy, and lower fertility rate (FR) [3]. Through FP programs, women have been given access to modern contraceptives, allowing them to better meet their fertility preferences and to avoid unwanted pregnancies and their potential complications [4-7]. Nonetheless, even in highly developed countries such as the U.S., unintended pregnancies remain high [8] and constitute a health and socioeconomic burden for women and their partners $[9,10]$. An obvious explanation is that there remain sociocultural barriers and perceived health risks associated with the use of new FP methods [11-13].

Previous research has shown that the main challenges facing women and preventing them from using modern contraceptive methods include the social pressure on women to prove their fertility immediately after marriage and the fear of side effects and negative consequences of modern FP methods [14-16]. In a recent qualitative study involving 42 focus group discussions with Jordanian and Syrian young couples, there was a strong belief among participants that most modern FP methods can have serious side effects that could harm women's health, including bleeding, hypertension, diabetes, foetal abnormalities, and cancer [17]. These concerns often drive married couples to use less effective but safer methods, such as withdrawal and condoms.

Women's personal experiences, knowledge, and perceptions of how a FP method might impact their quality of life (QoL) can influence FP decisions among women. While different FP methods may have varying degrees of impact on women's health-related quality of life (HRQoL) [18], there yet remains no consensus on the relationship between modern FP methods and the QoL of women. Most of the previous research has focused on either single or limited types of contraceptives and on only one or two QoL domains (i.e. sexual and psychological) [19-25]. For instance, it has been found that combined oral pills statistically decreased general well-being among healthy women and reduced three of the six dimensions of the Psychological General Well-Being Index (PGWBI), namely positive well-being, self-control, and vitality [19]. The authors concluded that even a modest reduction in general wellbeing could be of importance and could explain the high incompliance rate and irregular use of oral contraceptives
(OCs) [19]. Similarly, Sadatmahalleh et al. observed lower sexual function and QoL in women who had undergone tubal ligation than in women who had not, hence concluding that tubal ligation is not a safe FP method [26]. In contrast, intrauterine contraception has been shown to significantly improve HRQoL [20]. On the other hand, a recent systematic review by Worly et al. revealed that the available studies do not support a clear general association between progestin hormonal contraceptives and depression levels or incidence of depression diagnosis [25].

\section{Modern FP use among Jordanian women}

Although the Total Fertility Rate (TFR) in Jordan has declined steadily in recent decades to reach 3.5 in 2014, it has not changed significantly during the last few years [27]. Jordan has made some progress in meeting FP needs, such that the use of modern contraceptives increased by $15 \%$ from 1990 to 2012 [28]. However, the use of both modern and traditional FP methods has declined from 42 to $37 \%$, and from 19 to $14 \%$ between 2012 and 2017/2018, respectively. Among currently married women adopt a modern method of FP, the most commonly used method is the Intra Uterine Devices (IUDs) (56\%), followed by the pill (21\%), and the male condom $(13 \%)[28,29]$. The majority of modern contraceptive methods are accessible to women through the primary and comprehensive healthcare centers of the Ministry of Health. Oral contraceptives, Etonogestrel implants, Depo Provera contraceptive injections, male condoms, and IUDs are offered free of charge, and there are some facilities for female sterilization.

The process of choosing FP methods in Jordan is primarily influenced by the husband's decision and preference. About $85 \%$ of women currently using contraceptives report that the decision to use any FP method was a shared decision with the husband. Additionally, two thirds $(77 \%)$ of those who report not using any method say that the decision not to use was also a mutual decision with the husband, regardless of the education level. Women reported that they first discussed FP and the likelihood of using contraception with husbands, doctors and midwives, and friends. Oral contraceptives and IUDs were the two most common methods suggested by those advisers. When women decide to start using contraception, physicians were the most sought person to give advice as they are highly trusted by women [29, 30]. However, a lack of comprehensive understanding of the impact of modern FP methods continues to exist among Jordanian couples.

To date, research addressing the impact of different modern FP methods on women's QoL remains limited [31-34]. A study found that women who had undergone tubal ligation and those who used injectable contraceptive were less likely to have average or better physical 
HRQoL than women who used combined hormonal contraceptives and that women who used injectable contraceptive were also less likely to have average or better mental-HRQoL [18]. Another study showed that women who had undergone tubal ligation had significantly worse "physical functioning" but better "general health perceptions" than women whose partners used condoms [32].

To this end, the primary aim of this study was to examine the relationship between the use of common modern FP methods and QoL among Jordanian women. Also, identifying main predictors of QoL was another aim of the study.

\section{Methods}

\section{Study design and setting}

A cross-sectional household survey in two cities in Jordan was conducted from April to December 2017. Each city was stratified into 5 sectors based on geographical location and density of population. Housewives from each sector were visited in their houses, and working women who were unavailable during the morning were visited in the afternoon. An initial screening (shortstructured interviews) was first conducted to ensure that each woman was eligible and that she consented to participation in the study. The screening questions included information on age, health status, medical history, and whether the participant was pregnant. If the woman was eligible, the female interviewers explained to her the objectives of the study and assured her of the confidentiality of the interview before commencing with the interview itself. Talking about family planning methods is a socio-cultural sensitive issue in Jordan, especially among young women. Many Jordanian women usually feel embarrassed to discuss family planning methods, and this led to a low response rate of around $15 \%$ of the household survey. For this reason, non-pregnant women who visited the obstetrics and gynecology clinic of King Abdullah University Hospital (KAUH) for contraceptive advice and follow-up consultations were also included. KAUH is a teaching referral hospital that provides a wide range of services. Ethical approval was sought and obtained from the Institutional Research Committee of Jordan University of Science and Technology. After full clarification of the study aims and ethical considerations, any woman who showed interest in participating and gave consent was included. Since contraception is a culturally sensitive issue in Jordan, willingness to participate voluntarily was a main inclusion criterion, and thus, a convenience sampling method was applied.

\section{Data collection and measures}

According to the latest national survey, about $30 \%$ of Jordanian women discontinued using the method within
12 months. The most common reason for discontinuations was the desire to become pregnant (54\%), followed by complaints that the method was inconvenient to use (12\%) [29]. Consequently, non-pregnant women of child bearing age (18-49) who had been using a modern contraceptive method (the same method) for the past year were interviewed through face-to-face structured interviews. Three female researchers were trained to conduct the interviews, where each one was responsible for conducting the interviews in a particular geographical location/area. A structured interview questionnaire guide (in Arabic) composed of four main sections was used for data collection. The socio-demographic data form included questions about the woman's age, number of living children, age at marriage, and religion. The first section also included statements on socioeconomic status, including educational attainment, employment class, monthly income, housing tenure, and the number of dependents. The second section included questions on birth spacing and on the type, duration of use, and previous information of the modern FP methods used, as well as previous knowledge of their side effects. Another questionnaire, namely the Arabic version of World Health Organization Quality of Life-BREF (WHOQOLBREF), was used as the measuring instrument for QoL in the current study.

The WHOQOL-BREF is a cross cultural, well validated, generic questionnaire that has been employed in various clinical conditions in different countries around the globe [31, 35-37]. According to the WHO report [38], the WHOQOL-BREF serves as an adequate alternative instrument to the evaluation of domain profiles using WHOQOL-100. With a total of 26 questions, the instrument contains four domains, namely physical health, psychological, social relationships, and environment. These questions use a five point Likert response scale. There are also two questions in the WHOQOLBREF that are evaluated separately; the first question asks about the participant's overall perception of their QoL and the second about the participant's overall perception of their health [39].

While the WHOQOL-BREF examines respondents' perceived QoL and can thus provide an overview of the participant's perceived effects of disease and health interventions, it is not supposed to act as an objective tool for measuring symptoms and disability. Therefore, the WHOQOL is "an assessment of a multi-dimensional concept incorporating the individual's perception of health status, psycho-social status, and other aspects of life." [38].

As per the WHO guidelines, the mean score of questions within each domain is used to calculate the score of the domain; means are multiplied by 4 and subsequently transformed into a 0 to 100 scale [38]. The 
domain scores are comparable with the scores used in the WHOQOL-100, and the mean score in each domain reflects women's perceptions of and satisfaction with each aspect of their quality of life. The higher the score, the better the response. A permission to use the WHOQOL-BREF was obtained from the Information, Evidence, and Research (IER) Department / The World Health Organization, Geneva. The last section of the interview guide contained questions about side effects of modern FP methods and bleeding patterns. At the end of each interview, the participating woman was asked to read and check the filled data for accuracy, and then she was voluntarily asked to sign the filled questionnaire. Each interview lasted about $20 \mathrm{~min}$.

\section{Data analysis}

Data was analyzed using $\mathrm{R}$ Statistical Computing Software version 3.4.3 (R Foundation for Statistical Computing, Vienna, Austria). Descriptive statistics including means, standard deviations, and percentages for collected data are presented. QoL scores for the four domains were calculated individually for each participating woman, and then the total scores for each domain were summarized. Finally, predictors of quality of life scores were identified through multiple linear regression and stepwise selection. A 5\% significance level was assumed throughout the analysis.

\section{Results}

Five hundred forty-eight women aged between 18 and 49 (mean age 34.7, SD 6.14) participated in the study. The participating women had been using a FP method for an average of 5.17 (SD 2) years, discontinuously. The results show that the average time from marriage to first pregnancy was 3.31 (SD 2.36) months, and the average number of children per family was 3.79 (SD 1.85). The mean duration of spacing between births was 2.78 (SD 1.17) years. Detailed information on the participants is provided in Table 1. Around one third of the participants $(35.6 \%)$ had a graduate or post-graduate academic degree, around $33 \%$ had a family income less than 400 JDs, and 58.5\% resided in rented accommodation. In Jordan, there is no clear official definition of the poverty line. However, when taking into consideration the latest available data and reports, an estimated family income of (JD400) per month can be considered as the poverty line $[40,41]$. In accordance with the national figures, about $9.5 \%$ of the participants were smokers [42], and only $11.86 \%$ were employed [43]. The results also show that about $9.52 \%$ of the participating women used a FP method without a doctor's prescription. Unfortunately, about $80.66 \%$ of the women had no previous knowledge of the possible side effects of using FP methods, and only $46.72 \%$ of them had had enough information about FP methods before starting to use them.

As for the types of contraceptive methods used, $41.61 \%$ of the participants used IUDs, $23.18 \%$ used oral contraceptives, $14.78 \%$ used implant and injectable hormonal contraceptives, $6.2 \%$ used sterilization, and $14.23 \%$ reported that their husbands used condoms as a FP method. Only about 50\% rated their QoL as being good or very good and about $40.88 \%$ were satisfied with their health status.

Women's responses to QoL related questions of the WHOQOL-BREF tool are summarized in Table 2. Based on these responses, scores for the four QoL domains, namely physical health, psychological, social relationships, and environment, were calculated for each woman. A summary of these domain scores, along with the FP method used, is shown in Table 3. From highest to lowest, average scores for the social relationships, psychological, physical health, and environmental domains were 13.32, $12.97,12.94$, and 12.81, respectively, on a scale of 4-20. Women who used IUDs had the highest QoL average score for the physical health domain, while those whose husbands used condoms had a much higher average QoL scores for the psychological, social relationships, and environment domains than other women's groups.

Multiple linear regression in combination with the stepwise selection method was used to identify the significant predictors of the domain scores and to identify the effect of FP methods on the QoL of the participating women. As the effect of the FP method used might be confounded with other variables such as the socioeconomic status and age, all collected variables were used in the regression model to adjust for the effects of the FP methods. Significant predictors along with the model coefficients for the four domains are shown in Table 4. Multicollinearity among used predictors was assessed through variance inflation factors (VIF). Values for VIF were all less than 10 indicating no multicollinearity issues. Significant predictors of physical health scores were level of education, income, residency, employment status, birth spacing, side effects, type of contraception, quality of life rating, and satisfaction with health. In comparison to women who used OCs, women who used IUDs for family planning had the highest probability of good physical health scores $(\beta=1.29)$, followed by women who use implants and injectable hormonal contraceptives $(\beta=0.66)$, whereas those who used sterilizations were more likely to score low in the physical health score $(\beta=-0.99)$.

Level of education, number of children, birth spacing, side effects, contraceptive method, and QoL rating were the significant predictors of the psychological domain scores. Similar to the physical health scores, a negative effect was observed for sterilization $(\beta=-2.34)$, while 
Table 1 Summary of participants' information

\begin{tabular}{|c|c|c|c|c|c|c|c|}
\hline Factor & Categories & $\begin{array}{l}\text { Oral } \\
\text { contraceptives }\end{array}$ & IUD & $\begin{array}{l}\text { Implant and injectable hormonal } \\
\text { contraceptives }\end{array}$ & Sterilization & $\begin{array}{l}\text { Male } \\
\text { condoms }\end{array}$ & Total \\
\hline \multirow[t]{5}{*}{ Education level } & Illiterate & $0.78 \%$ & $2.19 \%$ & $2.46 \%$ & 0 & $7.69 \%$ & $2.55 \%$ \\
\hline & Primary school & $22.04 \%$ & $18.42 \%$ & $12.34 \%$ & $17.64 \%$ & $19.23 \%$ & $18.43 \%$ \\
\hline & Secondary school & $37.79 \%$ & $35.52 \%$ & $35.8 \%$ & $17.64 \%$ & $34.61 \%$ & $34.85 \%$ \\
\hline & High school & $7.08 \%$ & $8.33 \%$ & $7.40 \%$ & $20.58 \%$ & $8.97 \%$ & $8.75 \%$ \\
\hline & $\begin{array}{l}\text { Graduate/Post } \\
\text { Graduate }\end{array}$ & $32.28 \%$ & $35.52 \%$ & $41.97 \%$ & $29.14 \%$ & $29.48 \%$ & $35.58 \%$ \\
\hline \multirow[t]{2}{*}{ Income } & Less than $400 \mathrm{JD}$ & $35.43 \%$ & $27.19 \%$ & $35.80 \%$ & $41.17 \%$ & $30.76 \%$ & $32.52 \%$ \\
\hline & More than 400 JD & $62.20 \%$ & $70.61 \%$ & $60.49 \%$ & $58.82 \%$ & $65.38 \%$ & $59.85 \%$ \\
\hline \multirow[t]{3}{*}{ Residency } & Rent & $58.26 \%$ & $54.38 \%$ & $69.13 \%$ & $67.64 \%$ & $56.41 \%$ & $58.57 \%$ \\
\hline & Owned & $40.94 \%$ & $44.29 \%$ & $28.39 \%$ & $20.58 \%$ & $38.46 \%$ & $38.86 \%$ \\
\hline & Other & $0.78 \%$ & $1.31 \%$ & $2.46 \%$ & $11.76 \%$ & $3.84 \%$ & $2.37 \%$ \\
\hline \multirow[t]{2}{*}{ Smoking } & No & $88.97 \%$ & $91.6 \%$ & $96.29 \%$ & $85.29 \%$ & $85.89 \%$ & $91.61 \%$ \\
\hline & Yes & $11.02 \%$ & $8.33 \%$ & $3.70 \%$ & $14.70 \%$ & $14.10 \%$ & $9.49 \%$ \\
\hline \multirow[t]{2}{*}{ Employment status } & Employed & $10.23 \%$ & $7.45 \%$ & $18.51 \%$ & $20.58 \%$ & $26.92 \%$ & $11.86 \%$ \\
\hline & Unemployed & $89.76 \%$ & $92.54 \%$ & $81.48 \%$ & $79.41 \%$ & $73.07 \%$ & $88.13 \%$ \\
\hline \multirow[t]{2}{*}{ Prescribed FP } & No & $10.24 \%$ & $10.13 \%$ & $9.88 \%$ & $8.82 \%$ & $6.49 \%$ & $9.52 \%$ \\
\hline & Yes & $89.76 \%$ & $89.87 \%$ & $90.12 \%$ & $91.18 \%$ & $93.51 \%$ & $90.48 \%$ \\
\hline \multirow{2}{*}{$\begin{array}{l}\text { Prior knowledge of side } \\
\text { effects }\end{array}$} & Yes & $11.81 \%$ & $22.81 \%$ & $25.93 \%$ & $20.59 \%$ & $14.1 \%$ & $19.34 \%$ \\
\hline & No & $88.19 \%$ & $77.1 \% 9$ & $74.07 \%$ & $79.41 \%$ & $85.9 \%$ & $80.66 \%$ \\
\hline \multirow{2}{*}{$\begin{array}{l}\text { Had enough information } \\
\text { about FP }\end{array}$} & Yes & $46.46 \%$ & $44.3 \%$ & $54.32 \%$ & $41.18 \%$ & $48.72 \%$ & $46.72 \%$ \\
\hline & No & $53.54 \%$ & $55.7 \%$ & $45.68 \%$ & $58.82 \%$ & $51.28 \%$ & $53.28 \%$ \\
\hline \multirow[t]{5}{*}{ Noticed bleeding } & Always & $11.11 \%$ & $1.32 \%$ & $13.75 \%$ & 0 & $8.97 \%$ & $6.42 \%$ \\
\hline & Very often & $35.71 \%$ & $17.62 \%$ & $42.5 \%$ & $20.59 \%$ & $33.33 \%$ & $27.89 \%$ \\
\hline & Quite often & $33.33 \%$ & $25.11 \%$ & $36.25 \%$ & $17.65 \%$ & $26.92 \%$ & $28.44 \%$ \\
\hline & Seldom & $11.9 \%$ & $27.31 \%$ & $5 \%$ & $20.59 \%$ & $17.95 \%$ & $18.72 \%$ \\
\hline & Never & $7.94 \%$ & $28.63 \%$ & $2.5 \%$ & 41.18 & $12.82 \%$ & $18.53 \%$ \\
\hline \multirow[t]{5}{*}{ Self-reported QoL } & Very poor & $1.57 \%$ & $1.75 \%$ & $4.94 \%$ & $47.06 \%$ & $5.13 \%$ & $5.47 \%$ \\
\hline & Poor & $3.15 \%$ & $4.82 \%$ & $4.94 \%$ & $32.35 \%$ & $11.54 \%$ & $7.12 \%$ \\
\hline & Neither poor nor good & $48.82 \%$ & $34.65 \%$ & $43.21 \%$ & $5.88 \%$ & $34.62 \%$ & $37.41 \%$ \\
\hline & Good & $35.43 \%$ & $44.3 \%$ & $30.86 \%$ & $5.88 \%$ & $38.46 \%$ & $37.04 \%$ \\
\hline & Very good & $11.02 \%$ & $14.47 \%$ & $16.05 \%$ & $8.82 \%$ & $10.26 \%$ & $12.96 \%$ \\
\hline \multirow{5}{*}{$\begin{array}{l}\text { Self-reported health } \\
\text { satisfaction }\end{array}$} & Very dissatisfied & $1.57 \%$ & $3.07 \%$ & $1.23 \%$ & $38.24 \%$ & $5.13 \%$ & $4.93 \%$ \\
\hline & Dissatisfied & $14.96 \%$ & $10.53 \%$ & $18.52 \%$ & $26.47 \%$ & $14.1 \%$ & $14.23 \%$ \\
\hline & $\begin{array}{l}\text { Neither satisfied nor } \\
\text { dissatisfied }\end{array}$ & $40.94 \%$ & $46.05 \%$ & $33.33 \%$ & $26.47 \%$ & $33.33 \%$ & $39.96 \%$ \\
\hline & Satisfied & $42.52 \%$ & $36.4 \%$ & $46.91 \%$ & $5.88 \%$ & $35.9 \%$ & $37.41 \%$ \\
\hline & Very satisfied & 0 & $3.95 \%$ & 0 & $2.94 \%$ & $11.54 \%$ & $3.47 \%$ \\
\hline
\end{tabular}

positive effects were observed for IUDs $(\beta=0.05)$ and male condoms $(\beta=2.62)$. For the social relationships domain, age, income, occupation, birth spacing, contraceptive method, and satisfaction with health status were identified as the significant predictors. In comparison to women who used OCs, women who used IUDs $(\beta=$ 0.24 ) or implants and injectable hormonal contraceptives $(\beta=0.32)$ and those whose husbands used condoms $(\beta=$ 2.11 ) were more likely to have better scores, while those who used sterilization were more likely to score low in the social relationship $(\beta=-2.63)$. Finally, age, income, smoking, occupation, period of contraceptive use, birth spacing, contraceptive method, quality of life rating, and satisfaction with health status were the significant 
Table 2 Percentages of participants' responses for the quality of life related questions

\begin{tabular}{|c|c|c|c|c|c|c|}
\hline \multicolumn{2}{|c|}{ Question } & \multirow{2}{*}{$\begin{array}{l}\text { Not at all (\%) } \\
11.9\end{array}$} & \multirow{2}{*}{$\begin{array}{l}\text { A little } \\
(\%) \\
31.8\end{array}$} & \multirow{2}{*}{$\begin{array}{l}\text { A moderate } \\
\text { amount } \\
(\%) \\
42.0\end{array}$} & \multirow{2}{*}{$\begin{array}{l}\text { Very much } \\
(\%) \\
10.0\end{array}$} & \multirow{2}{*}{$\begin{array}{l}\text { An extreme } \\
\text { amount } \\
\text { (\%) } \\
4.4\end{array}$} \\
\hline Q3 & $\begin{array}{l}\text { To what extent do you feel that physical pain prevents you } \\
\text { from doing what you need to do? }\end{array}$ & & & & & \\
\hline Q4 & $\begin{array}{l}\text { How much do you need any medical treatment to function } \\
\text { in your daily life? }\end{array}$ & 12.4 & 30.3 & 37.6 & 12.6 & 7.1 \\
\hline Q5 & How much do you enjoy life? & 3.8 & 10.9 & 46.7 & 28.5 & 10.0 \\
\hline Q6 & To what extent do you feel your life to be meaningful? & 2.4 & 12.4 & 42.2 & 27.4 & 15.7 \\
\hline Q7 & How well are you able to concentrate? & 4.6 & 14.8 & 45.6 & 24.8 & 10.2 \\
\hline Q8 & How safe do you feel in your daily life? & 4.9 & 13.3 & 35.2 & 32.3 & 14.2 \\
\hline Q9 & How healthy is your physical environment? & 4.4 & 17.5 & 46.2 & 20.6 & 11.3 \\
\hline Q10 & Do you have enough energy for everyday life? & 8.2 & 27.9 & 42.0 & 16.1 & 5.8 \\
\hline Q11 & Are you able to accept your bodily appearance? & 4.6 & 14.1 & 39.8 & 26.8 & 14.8 \\
\hline Q12 & Have you enough money to meet your needs? & 2.0 & 17.2 & 48.4 & 24.8 & 7.7 \\
\hline Q13 & $\begin{array}{l}\text { How available to you is the information that you need in } \\
\text { your day-to-day life? }\end{array}$ & 5.3 & 20.7 & 38.0 & 28.4 & 7.5 \\
\hline \multirow[t]{2}{*}{ Q14 } & To what extent do you have the opportunity for leisure activities? & 6.6 & 18.8 & 41.1 & 23.0 & 10.6 \\
\hline & & Very bad & Bad & Okay & Good & Very good \\
\hline \multirow[t]{2}{*}{ Q15 } & How well are you able to get around? & 5.3 & 12.4 & 18.2 & 14.8 & 49.3 \\
\hline & & $\begin{array}{l}\text { Very } \\
\text { dissatisfied }\end{array}$ & Dissatisfied & $\begin{array}{l}\text { Neither satisfied nor } \\
\text { dissatisfied }\end{array}$ & Satisfied & Very satisfied \\
\hline Q16 & How satisfied are you with your sleep? & 7.5 & 19.5 & 23.9 & 35.0 & 14.1 \\
\hline Q17 & $\begin{array}{l}\text { How satisfied are you with your ability to perform your daily } \\
\text { living activities? }\end{array}$ & 11.9 & 16.6 & 19.7 & 30.8 & 21.0 \\
\hline Q18 & How satisfied are you with your capacity for work? & 23.5 & 21.0 & 26.8 & 24.3 & 4.4 \\
\hline Q19 & How satisfied are you with yourself? & 7.5 & 13.5 & 29.4 & 35.6 & 14.1 \\
\hline Q20 & How satisfied are you with your personal relationships? & 4.2 & 10.4 & 34.5 & 35.2 & 15.7 \\
\hline Q21 & How satisfied are you with your sex life? & 5.8 & 15.9 & 37.0 & 25.5 & 15.7 \\
\hline Q22 & How satisfied are you with the support you get from your friends? & 3.3 & 16.8 & 37.6 & 32.1 & 10.2 \\
\hline Q23 & How satisfied are you with the conditions of your living place? & 2.9 & 14.1 & 41.4 & 32.5 & 9.1 \\
\hline Q24 & How satisfied are you with your access to health services? & 10.8 & 25.0 & 34.7 & 20.6 & 8.9 \\
\hline \multirow[t]{2}{*}{ Q25 } & How satisfied are you with your transport? & 5.8 & 15.7 & 30.1 & 27.6 & 20.8 \\
\hline & & Always & Very often & Quite often & Seldom & Never \\
\hline Q26 & $\begin{array}{l}\text { How often do you have negative feelings such as blue mood, } \\
\text { despair, anxiety, depression? }\end{array}$ & 14.1 & 17.3 & 27.7 & 20.3 & 20.6 \\
\hline
\end{tabular}

Table 3 Summary of domain scores. Statistics are based on 4-20 scale

\begin{tabular}{|c|c|c|c|c|c|c|c|c|}
\hline \multirow[t]{2}{*}{ FP method } & \multicolumn{2}{|c|}{ Physical health } & \multicolumn{2}{|c|}{ Psychological } & \multicolumn{2}{|c|}{ Social relationships } & \multicolumn{2}{|c|}{ Environment } \\
\hline & Mean & SD & Mean & SD & Mean & SD & Mean & SD \\
\hline OCPs & 12.21 & 2.56 & 12.70 & 1.83 & 13.09 & 2.81 & 12.32 & 2.00 \\
\hline IUD & 13.77 & 1.90 & 12.89 & 2.14 & 13.32 & 3.03 & 13.11 & 2.05 \\
\hline Implant and injectable hormonal contraceptives & 12.83 & 1.94 & 12.69 & 2.12 & 13.32 & 2.44 & 12.24 & 2.06 \\
\hline Sterilization & 11.58 & 1.96 & 10.35 & 1.82 & 10.63 & 3.22 & 9.71 & 1.90 \\
\hline Male condoms & 12.42 & 2.35 & 15.30 & 2.26 & 15.57 & 2.78 & 14.70 & 2.46 \\
\hline Overall & 12.94 & 2.26 & 12.97 & 2.29 & 13.42 & 3.06 & 12.81 & 2.37 \\
\hline
\end{tabular}


Table 4 Predictors of the four QoL domains

\begin{tabular}{|c|c|c|c|c|}
\hline \multirow{2}{*}{$\begin{array}{l}\text { 1- Predictors of the physical health } \\
\text { domain scores. }\end{array}$} & & $\beta$ & $95 \% \mathrm{Cl}$ & $p$ \\
\hline & (Intercept) & 11.78 & $9.58-13.98$ & $<.001$ \\
\hline \multirow[t]{4}{*}{ Education } & Primary school & 2.81 & $0.79-4.84$ & 0.007 \\
\hline & Secondary school & 0.37 & $-1.43-2.16$ & 0.688 \\
\hline & Diploma & 1.48 & $-0.07-3.03$ & 0.061 \\
\hline & Bachelor's degree & 0.66 & $-1.00-2.33$ & 0.435 \\
\hline Income & More than $400 \mathrm{JD}$ & 0.73 & $0.35-1.11$ & $<.001$ \\
\hline \multirow[t]{2}{*}{ Residency } & Owned & 0.18 & $-0.20-0.57$ & 0.347 \\
\hline & Other & -1.1 & $-2.24-0.04$ & 0.059 \\
\hline Employment status & Employed & 0.79 & $0.17-1.42$ & 0.012 \\
\hline Birth spacing & & -0.16 & $-0.31--0.01$ & 0.04 \\
\hline \multirow[t]{4}{*}{ Experienced side effect after using FP } & Very often & 0.42 & $-0.14-0.97$ & 0.141 \\
\hline & Quite often & 0.46 & $-0.08-0.99$ & 0.092 \\
\hline & Seldom & 1.08 & $0.45-1.72$ & $<.001$ \\
\hline & Never & 0.76 & $0.11-1.42$ & 0.023 \\
\hline \multirow[t]{4}{*}{ Type of contraception used } & IUD & 1.29 & $0.79-1.79$ & $<.001$ \\
\hline & $\begin{array}{l}\text { Implant and injectable } \\
\text { hormonal contraceptives }\end{array}$ & 0.66 & $0.08-1.24$ & 0.026 \\
\hline & Sterilization & -0.99 & $-1.97-0.00$ & 0.05 \\
\hline & Male condoms & 0.1 & $-0.51-0.71$ & 0.746 \\
\hline \multirow[t]{4}{*}{ Self-reported QoL } & Poor & -1.39 & $-2.44--0.35$ & 0.009 \\
\hline & Neither poor nor good & -0.82 & $-1.74-0.10$ & 0.079 \\
\hline & Good & -0.11 & $-1.03-0.81$ & 0.816 \\
\hline & Very good & 0 & $-1.00-1.00$ & 0.997 \\
\hline \multirow[t]{4}{*}{ Self-reported health satisfaction } & Dissatisfied & -1.15 & $-2.12--0.17$ & 0.021 \\
\hline & Neither satisfied nor dissatisfied & -1.17 & $-2.10--0.23$ & 0.015 \\
\hline & Satisfied & -0.75 & $-1.70-0.20$ & 0.123 \\
\hline & Very satisfied & -1.73 & $-3.10--0.36$ & 0.013 \\
\hline$R^{2}$ & & 0.264 & & \\
\hline $\begin{array}{l}\text { 2- Predictors of the psychological } \\
\text { domain scores }\end{array}$ & & $\beta$ & $95 \% \mathrm{Cl}$ & $p$ \\
\hline (Intercept) & & 12.49 & $10.55-14.42$ & $<.001$ \\
\hline Age & & -0.04 & $-0.08-0.00$ & 0.054 \\
\hline \multirow[t]{4}{*}{ Education } & Primary school & 2.52 & $0.55-4.49$ & 0.012 \\
\hline & Secondary school & 2.31 & $0.58-4.04$ & 0.009 \\
\hline & Diploma & 1.8 & $0.31-3.29$ & 0.018 \\
\hline & Bachelor's degree & 1.53 & $-0.14-3.21$ & 0.072 \\
\hline Number of children & & 0.34 & $0.07-0.60$ & 0.012 \\
\hline Time of first pregnancy & & -0.05 & $-0.12-0.02$ & 0.159 \\
\hline Birth spacing & & -0.22 & $-0.37--0.07$ & 0.003 \\
\hline \multirow[t]{4}{*}{ Experienced side effect after using FP } & Very often & 0.29 & $-0.25-0.83$ & 0.287 \\
\hline & Quite often & 0.28 & $-0.24-0.79$ & 0.289 \\
\hline & Seldom & 0.05 & $-0.56-0.65$ & 0.879 \\
\hline & Never & -0.61 & $-1.25-0.02$ & 0.059 \\
\hline Type of contraception used & IUD & 0.05 & $-0.44-0.54$ & 0.849 \\
\hline
\end{tabular}


Table 4 Predictors of the four QoL domains (Continued)

\begin{tabular}{|c|c|c|c|c|}
\hline & Implant and injectable hormonal contraceptives & -0.14 & $-0.71-0.43$ & 0.635 \\
\hline & Sterilization & -2.34 & $-3.25--1.43$ & $<.001$ \\
\hline & Male condoms & 2.62 & $2.03-3.21$ & $<.001$ \\
\hline \multirow[t]{4}{*}{ Self-reported QoL } & Poor & -0.08 & $-1.06-0.90$ & 0.87 \\
\hline & Neither poor nor good & -0.57 & $-1.45-0.30$ & 0.198 \\
\hline & Good & -0.14 & $-1.03-0.74$ & 0.751 \\
\hline & Very good & 0.2 & $-0.75-1.15$ & 0.684 \\
\hline$R^{2}$ & & 0.312 & & \\
\hline $\begin{array}{l}\text { 3- Predictors of the social relationships } \\
\text { domain scores }\end{array}$ & & $\beta$ & $95 \% \mathrm{Cl}$ & $p$ \\
\hline (Intercept) & & 16.21 & $14.10-18.32$ & $<.001$ \\
\hline Age & & -0.05 & $-0.10--0.00$ & 0.039 \\
\hline Income & More than $400 \mathrm{JD}$ & 0.53 & $-0.03-1.09$ & 0.062 \\
\hline Employment status & Employed & 0.71 & $-0.01-1.43$ & 0.053 \\
\hline Birth spacing & & -0.24 & $-0.44--0.03$ & 0.024 \\
\hline \multirow[t]{4}{*}{ Type of contraception used } & IUD & 0.24 & $-0.40-0.88$ & 0.465 \\
\hline & Implant and injectable hormonal contraceptives & 0.32 & $-0.49-1.13$ & 0.439 \\
\hline & Sterilization & -2.63 & $-3.80--1.46$ & $<.001$ \\
\hline & Male condoms & 2.11 & $1.28-2.94$ & $<.001$ \\
\hline \multirow[t]{4}{*}{ Self-reported health satisfaction } & Dissatisfied & -1.54 & $-2.87--0.22$ & 0.022 \\
\hline & Neither satisfied nor dissatisfied & -1.49 & $-2.75--0.24$ & 0.02 \\
\hline & Satisfied & -0.86 & $-2.12-0.41$ & 0.185 \\
\hline & Very satisfied & 1.12 & $-0.71-2.95$ & 0.23 \\
\hline$R^{2}$ & & 0.179 & & \\
\hline $\begin{array}{l}\text { 4- Predictors of the environmental } \\
\text { domain scores. }\end{array}$ & & $\beta$ & $95 \% \mathrm{Cl}$ & $p$ \\
\hline (Intercept) & & 14.67 & $12.85-16.49$ & $<.001$ \\
\hline Age & & -0.04 & $-0.09--0.00$ & 0.049 \\
\hline Income & More than $400 \mathrm{JD}$ & 0.98 & $0.58-1.38$ & $<.001$ \\
\hline Smoking & Yes & 0.48 & $-0.15-1.10$ & 0.135 \\
\hline Employment status & Employed & 0.94 & $0.41-1.48$ & $<.001$ \\
\hline Duration of using FP & & 0.1 & $-0.02-0.22$ & 0.113 \\
\hline Birth spacing & & -0.15 & $-0.30--0.01$ & 0.04 \\
\hline \multirow[t]{4}{*}{ Type of contraception used } & IUD & 0.7 & $0.24-1.15$ & 0.003 \\
\hline & Implant and injectable hormonal contraceptives & -0.14 & $-0.71-0.44$ & 0.641 \\
\hline & Sterilization & -3.21 & $-4.16--2.27$ & $<.001$ \\
\hline & Male condoms & 2.08 & $1.49-2.67$ & $<.001$ \\
\hline \multirow[t]{4}{*}{ Self-reported QoL } & Poor & -0.37 & $-1.40-0.65$ & 0.476 \\
\hline & Neither poor nor good & -0.71 & $-1.62-0.20$ & 0.127 \\
\hline & Good & -0.05 & $-0.96-0.86$ & 0.917 \\
\hline & Very good & 0.65 & $-0.33-1.62$ & 0.193 \\
\hline \multirow[t]{4}{*}{ Self-reported health satisfaction } & Dissatisfied & -1.15 & $-2.11--0.19$ & 0.019 \\
\hline & Neither satisfied nor dissatisfied & -1.4 & $-2.32--0.49$ & 0.003 \\
\hline & Satisfied & -1.39 & $-2.33--0.46$ & 0.004 \\
\hline & Very satisfied & -0.54 & $-1.87-0.79$ & 0.424 \\
\hline$R^{2}$ & & 0.312 & & \\
\hline
\end{tabular}


predictors of the environmental domain scores. For the environmental domain, women who used IUDs $(\beta=0.7)$ and those whose husbands used condoms $(\beta=2.08)$ as FP methods reported higher scores than women who used OCs. However, women who had received permanent sterilization had the worst environmental scores among all of the family planning methods $(\beta=-3.21)$.

To further investigate the effect of FP methods on QoL, multinomial logistic regression was used to identify significant predictors of side effects frequency, bleeding frequency, QoL rating perceptions, and satisfaction with health status rating. Identified significant predictors are shown in Table 5. Results indicate that the type of contraceptive method used was a key predictor of side effects, bleeding, QoL perception, and health satisfaction. Table 5 shows that women who had undergone sterilization reported the highest probability of frequent side effects while those whose husbands used condoms had a high probability of experiencing rare or no side effects. Women who used implant and injectable hormonal contraceptives had a very low probability of never experiencing side effects. Similarly, women who had undergone sterilization and those who used IUDs had the highest probability of bleeding. Results also show that women who used IUDs had the highest probability of good/very good quality of life perceptions while sterilized women had the lowest probability of good/very good perceptions.

Women who used IUDs or implant and injectable hormonal contraceptives and women whose husbands used condoms had the highest level of health satisfaction, while women who used oral pills and sterilization had the lowest health satisfaction.

\section{Discussion}

From social, psychological, and health-related viewpoints, FP methods are of great importance in women's lives $[44,45]$. This study investigated the relationship between the use of common modern FP methods and QoL domains among Jordanian women. In line with the latest Jordanian national survey 2017/2018 [29], our findings observed that the most commonly used modern FP method is the IUDs, followed by OCs. The findings also revealed that about a tenth of Jordanian women of reproductive age had previously used a modern FP method without medical prescriptions or advice. Furthermore, more than half of the participating women did not have enough previous information about modem FP methods. It has been found recently that the percentage of Jordanian women who were not informed about side effects of modern FP methods ranged between 20 to 39\% among various methods including pills, female sterilization, IUD, implants and injectables [29]. Nevertheless, women who intend to use modern FP methods have the right to know the potential side effects and negative consequences of these methods, including emotional, psychological, physiological, and sexual consequences.

Most women in developing countries, including Jordan, prefer to get pregnant immediately after marriage [46-49], and hence an average time from marriage till first pregnancy of only 3.31 months was observed among the participating women. Often, this short waiting period is a result of social pressure and reflects the tendency among young women to prove their fertility immediately after marriage, regardless of whether they are physically and emotionally prepared [50-52]. It has also been argued that, in some cases, Jordanian women may use their ability to get pregnant soon after marriage as a way to raise their status and power among their in-laws. This provides insight on why FP methods are usually only considered after the first child has been born [53, 54].

Based on the WHOQOL-BREF scale, the overall mean (SD) scores of the four domains were found to be average, with the social relationships mean score being slightly higher than the other domains and the environmental domain being slightly lower.

Our results also revealed that women who used IUDs and women whose husbands used condoms as FP methods had better QoL in the four domains (physical health, psychological health, social relationships, and environment) than women who used OCs. In line with our findings, a recent prospective observational multicenter study conducted in Spain found that the Levonorgestrel intrauterine system (LNG-IUS) has a positive impact in terms of health related QoL. The results were obtained through conducting a SEC-QoL questionnaire at baseline (at the start of the LNG-IUS use) and 12 months afterwards. The study demonstrated improved scores in all domains of the SEC-QoL, including the social, menstrual symptoms, breast symptoms, psychological, and sexual domains. Beside its efficacy as a FP method, LNG-IUS provided women with additional non-contraceptive benefits (i.e. reduction in bleeding and menstrual pain) [21]. Similar results were reported by Singh from India [55].

Though IUDs may cause mild side effects which may interfere with a woman's QoL, such as irregular bleeding, amenorrhea, and hormonal disturbances [56, 57], women who used IUDs and those whose husbands used condoms were nonetheless more satisfied than women who used OCs and sterilization. The use of female condoms is uncommon among Jordanian women, and $14.23 \%$ of the participating women in our study reported that their husbands used male condoms. Loss of sexual function and pleasure (among both males and females) is reported as being the most devastating side effect of using condoms $[58,59]$.

In consistence with our results, a recent randomized, double-blind, placebo-controlled trial which assessed the 
Table 5 Predictors of side effect, bleeding frequency, quality of life rating perceptions, and health satisfaction

\begin{tabular}{|c|c|c|c|c|c|}
\hline $\begin{array}{l}\text { Predictors of side } \\
\text { effects frequency }\end{array}$ & & $\begin{array}{l}\text { Very often } \\
\text { coefficients ( } \beta \text { ) }\end{array}$ & $\begin{array}{l}\text { Quite often } \\
\text { coefficients ( } \beta \text { ) }\end{array}$ & Seldom coefficients ( $\beta$ ) & $\begin{array}{l}\text { Never } \\
\text { coefficients ( } \beta \text { ) }\end{array}$ \\
\hline (Intercept) & & -0.235 & -0.206 & -1.564 & -2.375 \\
\hline \multirow{4}{*}{$\begin{array}{l}\text { Type of } \\
\text { contraception used }\end{array}$} & IUD & 0.037 & 1.752 & 2.969 & 3.953 \\
\hline & $\begin{array}{l}\text { Implant and injectable } \\
\text { hormonal contraceptives }\end{array}$ & 0.117 & -0.046 & 0.214 & -12.639 \\
\hline & Sterilization & 1.844 & 2.151 & 3.760 & 4.859 \\
\hline & Male condoms & 0.493 & 0.317 & 0.928 & 1.621 \\
\hline$R^{2}$ & & 0.096 & & & \\
\hline $\begin{array}{l}\text { Predictors of } \\
\text { bleeding frequency }\end{array}$ & & $\begin{array}{l}\text { Very often } \\
\text { coefficients ( } \beta \text { ) }\end{array}$ & $\begin{array}{l}\text { Quite often } \\
\text { coefficients ( } \beta \text { ) }\end{array}$ & $\begin{array}{l}\text { Seldom } \\
\text { coefficients ( } \beta \text { ) }\end{array}$ & $\begin{array}{l}\text { Never } \\
\text { coefficients ( } \beta \text { ) }\end{array}$ \\
\hline (Intercept) & & 0.767 & -0.400 & -0.683 & -1.422 \\
\hline Number of kids & & 0.200 & 0.660 & 0.360 & 0.497 \\
\hline \multirow{4}{*}{$\begin{array}{l}\text { Type of } \\
\text { contraception used }\end{array}$} & IUD & 1.321 & 1.673 & 2.754 & 3.221 \\
\hline & $\begin{array}{l}\text { Implant and injectable } \\
\text { hormonal contraceptives }\end{array}$ & -0.072 & -0.250 & -1.144 & -1.461 \\
\hline & Sterilization & 12.473 & 12.405 & 13.553 & 14.661 \\
\hline & Male condoms & 0.069 & -0.121 & 0.435 & 0.347 \\
\hline$R^{2}$ & & 0.079 & & & \\
\hline $\begin{array}{l}\text { Predictors of quality of } \\
\text { life rating perceptions }\end{array}$ & & $\begin{array}{l}\text { Poor } \\
\text { coefficients ( } \beta \text { ) }\end{array}$ & $\begin{array}{l}\text { Neither poor nor } \\
\text { good coefficients }(\beta)\end{array}$ & $\begin{array}{l}\text { Good } \\
\text { coefficients ( } \beta \text { ) }\end{array}$ & $\begin{array}{l}\text { Very good } \\
\text { coefficients ( } \beta \text { ) }\end{array}$ \\
\hline (Intercept) & & 4.291 & 6.855 & 6.681 & 0.992 \\
\hline Age & & -0.088 & -0.247 & -0.252 & -0.215 \\
\hline Occupation & Employed & 1.077 & 2.652 & 2.227 & -7.784 \\
\hline Number of children & & -1.334 & 0.516 & 0.492 & 0.088 \\
\hline \multirow{4}{*}{$\begin{array}{l}\text { Type of } \\
\text { contraception used }\end{array}$} & IUD & 0.351 & 0.107 & 0.608 & 0.751 \\
\hline & $\begin{array}{l}\text { Implant and injectable } \\
\text { hormonal contraceptives }\end{array}$ & -0.364 & -0.600 & -0.730 & -0.086 \\
\hline & Sterilization & -1.435 & -5.528 & -5.308 & -3.771 \\
\hline & Male condoms & 0.189 & -1.003 & -0.633 & -0.706 \\
\hline$R^{2}$ & & 0.135 & & & \\
\hline $\begin{array}{l}\text { Predictors of health } \\
\text { satisfaction rating }\end{array}$ & & $\begin{array}{l}\text { Dissatisfied } \\
\text { coefficients ( } \beta \text { ) }\end{array}$ & $\begin{array}{l}\text { Neither satisfied nor } \\
\text { dissatisfied coefficients ( } \beta \text { ) }\end{array}$ & $\begin{array}{l}\text { Satisfied } \\
\text { coefficients ( } \beta \text { ) }\end{array}$ & $\begin{array}{l}\text { Very satisfied } \\
\text { coefficients ( } \beta \text { ) }\end{array}$ \\
\hline (Intercept) & & 16.524 & 37.553 & 35.287 & 14.479 \\
\hline Age & & -0.119 & -0.218 & -0.112 & -0.268 \\
\hline \multirow[t]{4}{*}{ Education } & Primary school & -1.470 & 12.234 & -22.899 & 31.520 \\
\hline & Secondary school & 13.002 & -15.804 & -16.384 & -26.168 \\
\hline & Diploma & 12.966 & -14.480 & -14.109 & -14.464 \\
\hline & Bachelor's degree & 3.744 & 28.977 & 28.734 & 31.989 \\
\hline Income & More than 400 JD & 8.418 & 19.101 & 17.793 & 8.403 \\
\hline Number of children & & 1.164 & 1.145 & 1.227 & 0.398 \\
\hline Prescribed FP & Yes & -35.251 & -34.918 & -34.166 & -35.935 \\
\hline Years of using FP & & 0.133 & 0.113 & -0.115 & -0.300 \\
\hline
\end{tabular}


Table 5 Predictors of side effect, bleeding frequency, quality of life rating perceptions, and health satisfaction (Continued)

\begin{tabular}{|c|c|c|c|c|c|}
\hline \multirow{4}{*}{$\begin{array}{l}\text { Type of } \\
\text { contraception used }\end{array}$} & IUD & -0.933 & -0.098 & -0.689 & 36.992 \\
\hline & $\begin{array}{l}\text { Implant and injectable } \\
\text { hormonal contraceptives }\end{array}$ & -2.147 & -2.904 & -4.713 & 35.440 \\
\hline & Sterilization & 0.637 & 0.398 & 0.439 & 4.760 \\
\hline & Male condoms & -0.951 & -0.997 & -1.254 & 37.529 \\
\hline$R^{2}$ & & 0.158 & & & \\
\hline
\end{tabular}

influence of a first-choice combined oral contraceptive on general well-being and depressive symptoms found that contraceptive pills are associated with reduced general well-being, self-control, and vitality among healthy women [19]. Also, in Japan, it has been reported that OCs can worsen QoL if used for contraceptive purpose only [60]. On the other hand, other studies have reported that OCs have no negative impact on QoL, especially among first time users [61]. Zhao et al. conducted a prospective cohort study on rural women who used OCs and IUDs in a Chinese province; the study found that oral pills and IUDs could significantly improve overall QoL [62]. The mechanism behind reduction in well-being by the use of OCs among healthy women is yet to be discovered. However, the decrease in free testosterone level induced by OCs use and the direct progestin-induced central nervous system effect may be the underlying causes behind reduced well-being [19].

Our results observed that women who had been using implant and injectable hormonal contraceptives have better QoL in terms of the physical health and social relationships domains. Di Carlo et al. studied the impact of contraceptive implants on QoL and sexual function among Italian women. Their results showed that general QoL and physical role status of the women improved significantly after use of the implant and that the implant had some positive effect on sexual function [63]. In a review of evidence on implant contraindications, satisfaction, and rate of continuation, Amico et al. found that whilst irregular bleeding was the most common reason for discontinuation of the implant, most of the participating women reported an overall reduction in bleeding. Additionally, complications related to its use, insertion, and removal were rarely reported [64]. Consistently, the Short Form-36 QoL questionnaire was employed to evaluate the HRQoL changes associated with the use of the depot medroxyprogesterone acetate (DMPA) injection. Along with its contraceptive efficacy, DMPA was linked with improvement in perceived physical health and with no adverse effects on mental health and sexual function [65].

To identify predictors of QoL for women using FP methods, multiple linear regression models were fitted to the scores of the four QoL domains. Common QoL predictors such as education level, family income, employment status and place of living were also identified as predictors of QoL for women using FP methods. Significant method specific predictors were also identified including birth spacing, experiencing side effects, and type of modern FP method. Regression results indicated that the more frequent side effects, the lower physical health. Women who used IUD as their FP method had higher probability of better physical QoL scores compared to women who used other methods. Those whose husbands used male condoms have shown higher probability of better QoL scores for the psychological, social relationships and environmental domains.

The multiple linear regression analysis confirmed that, in comparison with women who used oral pills, women who had chosen permanent sterilization as their contraceptive method had the lowest QoL scores in all of the four domains. This is not surprising given the adverse effects and negative consequences that tubal ligation can have on women's overall wellbeing including psychological wellbeing and sense of productivity [66]. Similarly, Bahrami et al. adopted the (WHQOL-BREF) to compare the effects of different contraceptive methods on women's QoL and found that women who had used sterilization or (DMPA) injectable contraceptive had the lowest level of QoL [67]. The logistic regression analysis extended our findings and showed that women who had undergone permanent sterilization were more likely to experience side effects and vaginal bleeding than women who used OCs. On the other hand, women who had been using other types of modern FP methods had less likelihood of experiencing side effects and bleeding. A study conducted on women who performed tubal ligation method found that one third of women perceived tubal ligation as a risky procedure that should not be performed, whereas the majority of women indicated that they will not advise women to undergo this procedure [68]. Another study revealed that depression significantly increased after tubal ligation due to several factors including changes in self-image, fear of negative perceptions of other people, and most importantly the husband's negative attitude towards the procedure [69].

Because female sterilization or tubal ligation is a permanent contraceptive method which requires a one-time effort, some women still consider it to be the most convenient and effective FP method. In an Iranian study, 
many of the participating women stated that their main reason for choosing sterilization was to avoid the side effects of other contraceptive methods. However, the study results showed that the mean total scores of the SF-12 (Short Form Health Survey for evaluating QoL) and sexual function were significantly lower in the female sterilization group than in the non-sterilization group. $20 \%$ of the women in the sterilization group regretted their decision [26]. The main reason why these women had chosen permanent sterilization was that they felt fully satisfied with their family size [26]. However, the most widely known biological change after terminating fertility by tubal ligation is the post-sterilization syndrome, which comprises symptoms such as hormonal disturbance, menstrual irregularities, and vaginal bleeding [70-72]. This could explain why an increasing number of women are opting for reversible long-acting FP contraceptive methods rather than permanent tubal ligation [73].

Our multinomial logistic regression analysis showed that women who used IUDs and those who had undergone sterilization had a higher probability of experiencing vaginal bleeding than women who used OCs, yet women who used IUDs had the highest QoL (good/ very good). It has been argued that dissatisfaction due to bleeding profile is the most common reason for the discontinuation of a FP method [74]. Previous studies confirm that women who use IUDs report higher levels of satisfaction than users of other FP methods [75, 76]. Our analysis also demonstrated that women who used OCs and permanent sterilization were less satisfied with their health status than women who used other modern FP methods.

\section{Strengths and limitations}

The current study has some limitations that need to be acknowledged. First, the sample collected through a cross-sectional household design from only two cities in Jordan; in which all eligible women were chosen based on a convenience frame. The convenience selectivity of the sample limits the generalizability of the findings as there is a possibility that women who agreed to participate in this study differed from those who refused to do so. For example, women who declined participation could have been unwilling to talk about their experiences with using modern FP methods or share their perceptions for several reasons that could directly impact QoL. Regardless, the authors tried to increase representativeness of the sample by stratifying each city into 5 sectors based on geographical location and density of population. Future research need to conduct a national survey using a randomization process to increase representativeness of the findings to a wider population.
Second, QoL was self-reported, and this might have been associated with false reporting. As with all selfreporting surveys, there is always a chance with inaccurate answers that over-estimate patients' responses due to many reasons including courtesy bias [77], recall bias, and/or social desirability bias [77, 78]. However, the WHOQOL-BREF is a cross cultural, well validated, generic questionnaire that has been employed in various clinical conditions, including contraceptive use, in different countries worldwide [31, 35-37, 67]. Future studies need to use more objective measures to assess the impact of modern FP methods on women's QoL, preferably using a longitudinal design to draw causal inferences. Additionally, Further research need to design and/or use more relevant questionnaires that truly reflect and measure the real perceptions of QoL among users of modern FP methods taking into considerations medical history and overall health status of women. Nonetheless, the authors tried to cover some of these aspects in this study by asking women specific questions related to FP methods and QoL such as birth spacing, the type of method used, duration of use, and previous information of the modern FP methods used, as well as previous knowledge of their side effects.

Finally, although we faced several difficulties in recruiting participants and in data collection, this study can be considered the first study that attempted to explore the association between modern FP methods and women's QoL in Jordan.

\section{Implications}

The current study revealed that the overall average scores of the four domains of QoL were relatively low among women who were using modern FP methods. This raises an important question about whether Jordanian women who are not using modern FP methods have the same low level of QoL. A national household survey in Jordan found that females reported poorer HRQoL in comparison with their counterpart males [79]. Thus, more attention to this segment of the population need to be provided to improve all aspects of QoL associated with reproductive health, not merely those related to FP issues. Similarly, more research need to be undertaken to compare QoL levels among Jordanian women who use either modern or traditional FP methods versus those who do not in order to establish a baseline for overall HRQoL among Jordanian women in general. Simple QoL questions could be used by health care professionals as a screening tool for overall health at every medical contact. Our findings also call for establishing programs to assess QoL among women in Jordan and other Arab countries focusing on the potential impact of modern FP use on overall QoL. These programs should be widely available for women who need it the most across the country. 
However, women's decisions about the best FP options are highly affected by the method's efficacy, medical conditions, personal subjective experience with side effects or negative consequences, and the method's impact on QoL [16, 80-82]. Jordanian women have some misconceptions in regards to the use and side effects of modern FP methods. Even worse, lack of trust between women and healthcare providers donates to the high percentages of discontinuation and/or reluctance to seek alternatives [53]. Hence, women who intend to use modern FP methods have the right to know the potential side effects and negative consequences of these methods, including emotional, psychological, physiological, and sexual consequences. Health care professionals, especially trained midwives, need to be willing to spend enough time with women, especially at maternal and child health medical centers, to increase their awareness about the realistic benefits and side effects of each of the modern FP methods in order to change some women's misperceptions in this regard and ultimately increase their adoption of effective methods. This is of paramount importance in light of the findings of a recent national survey (2017/2018), which found that more than $60 \%$ of Jordanian women of childbearing age do not use any modern FP methods [29].

Further, the results of this study shed light on the importance of providing effective counselling and education on FP methods to women of reproductive age in Jordan, and underlines a need to provide couples with premarital counselling on FP and potential risks associated with early pregnancy, particularly in rural areas with high fertility rates. Importantly, clinicians need to use a patient-centred approach when providing counselling about all FP methods and support women's reproductive autonomy [64, 83]. This can be achieved by providing and securing various alternatives for women in regards to modern FP methods especially those that are perceived as well tolerated, highly effective in controlling blood loss, such as IUD and definitely a better alternative to hysterectomy for women with heavy menstrual bleeding [55].

The current study also found that women who used IUDs and women whose husbands used condoms were nonetheless more satisfied and had better QoL than women who used OCs and sterilization. Studies show that women who encounter complications while using a certain contraception method tend to have a lower QoL. Similarly, women who experienced side effects reported lower QoL scores than those who did not [84]. In the case of condom use, it is less likely that women experience side effects thus they tend to be more satisfied. Indicating a need for designing and implementing specific interventions, as a part of counseling practice, that teach women ways to cope with side effects and/or complications with various modern FP methods and evaluating the impact of these methods on both QoL and sexual life [84].

The relatively higher QoL scores observed among women whose husbands used condoms raising the issue of male participation in the use of contraception. A study conducted on male Muslims in a Ghanaian municipality concluded that condom is the most preferred and used modern contraceptive, suggesting that male Muslims need to be motivated to discuss FP with friends and wives, as well as programs should be developed to focus on involving men in the decision of FP with women [85]. This is very important given the fact that sociocultural factors could play a vital role in male involvement in FP. Therefore, social support, adequate information, and shared responsibility can all motivate men to have a more vital role in FP and use of certain contraceptive methods. Additionally, increasing literacy of reproductive health among men to improve their attitude and change their perceptions to better participate in reproductive health services is needed [86, 87]. Male participation in FP, in turn, enhances the status of women. A qualitative study on Iranian Muslim women revealed that participants felt more empowered when shared FP decisions were made with their male spouses based on agreement and support [88]. Consequently, more attention is needed to underpin strategies to encourage gender equity, shared decision making, shared responsibility and actual participation of men, empowering women, and to optimize worth of male participation [89].

\section{Conclusion}

Overall, participating women reported relatively poor quality of life particularly those using oral contraceptive bills in comparison to those who use IUDs and male condoms. When comparing QoL across different methods of modern contraceptives, we found that women who used oral contraceptives and those who had undergone permanent tubal sterilization were likely to have a lower level of QoL than users of IUDs and implant and injectable hormones and women whose husbands used condoms. Also, women who used oral contraceptives and women who had undergone tubal sterilization were less likely to be satisfied and had the highest probability of frequent bleeding. Being able to decide freely whether and when to have children is regarded as a fundamental reproductive health right and is strongly linked to women's health and quality of life.

\section{Abbreviations}

DMPA: Depot medroxyprogesterone acetate; FP: Family planning; FR: Fertility rate; HRQOL: Health-related quality of life; IER: Information, Evidence, and Research; IUDs: Intra Uterine Devices; JD: Jordanian Dinar; KAUH: King Abdullah University Hospital; LNG-IUS: Levonorgestrel intrauterine system; OCPs: Oral contraceptive pills; OCs: Oral contraceptives; PGWBI: Psychological General Well-Being Index; QoL: Quality of life; SD: Standard deviation; SEC- 
QOL: Spanish Society of Contraception Quality-Of-Life; SF-12: Short Form Health Survey for evaluating QoL; TFR: Total Fertility Rate; WHO: World Health Organization; WHOQOL-BREF: World Health Organization Quality of Life-BREF

\section{Acknowledgements}

We are very grateful to all participant women for their time and their cooperation in this research study.

\section{Authors' contributions}

MSA and HHH conceived the ideas of this study, participated in the study design, analysis and interpretation, as well as drafted and amended for the manuscripts. HAA and NAA carried out the statistical analysis and wrote the first draft of the results, they also contributed in data interpretation. DA, SM, SQ, and SD contributed in study design, data collection and interpretation of the results. Professor YSK critically revised the manuscript, he also had a significant contribution to analysis and interpretation of data. All co-authors read and approved the final draft.

\section{Funding}

Nothing to declare.

\section{Availability of data and materials}

The datasets generated and analysed during the current study are included in this manuscript article (Tables 1, 2, 3, 4 and 5).

\section{Ethics approval and consent to participate}

Ethical approval was sought and obtained from the Institutional Research Committee of Jordan University of Science and Technology. Also, a permission to use the WHOQOL-BREF was obtained from the Information, Evidence, and Research (IER) Department / The World Health Organization, Geneva.

After full clarification of the study aims and ethical considerations, any woman who showed interest in participating and gave consent was included. At the end of each interview, participating woman was asked to read and check the filled data for accuracy, and then she was voluntarily asked to sign the filled questionnaire.

\section{Consent for publication}

Not applicable.

\section{Competing interests}

The authors declare that they have no competing interests.

\section{Author details}

'Department of Health Management and Policy, Faculty of Medicine, Jordan University of Science and Technology, P.O.Box 3030, Irbid 22110, Jordan. ${ }^{2}$ Industrial Engineering, Faculty of Engineering, Jordan University of Science and Technology, P.O.Box (3030), Irbid 22110, Jordan. ${ }^{3}$ Allied Medical Sciences Department/Faculty of Applied Medical Sciences, Jordan University of Science and Technology, P.O.Box (3030), Irbid 22110, Jordan. ${ }^{4}$ Medical Education and Biostatistics, Department of Community Medicine, Public Health and Family Medicine, Jordan University of Science \& Technology, Irbid 22110, Jordan

Received: 30 January 2019 Accepted: 24 September 2019

\section{Published online: 15 October 2019}

\section{References}

1. United Nations Population Fund. Chapter VII. Reproductive rights and reproductive health. In: Programme of action. adopted at the International Conference on Population and Development, Cairo; 2004. https://www. unfpa.org/sites/default/files/event-pdf/PoA_en.pdf. Accessed 12 May 2018.

2. Prata N, Fraser A, Huchko MJ, Gipson JD, Withers M, Lewis S, et al. Women's empowerment and family planning: a review of the literature. J Biosoc Sci. 2017:49(6):713-43.

3. Upadhyay UD, Gipson JD, Withers M, Lewis S, Ciaraldi EJ, Fraser A, et al. Women's empowerment and fertility: a review of the literature. Soc Sci Med. 2014;115:111-20.

4. Sedgh $G$, Singh $\mathrm{S}$, Hussain R. Intended and unintended pregnancies worldwide in 2012 and recent trends. Stud Fam Plan. 2014;45(3):301-14.
5. Ezeh AC, Bongaarts J, Mberu B. Global population trends and policy options. Lancet. 2012:380(9837):142-8.

6. Tsui AO, McDonald-Mosley R, Burke AE. Family planning and the burden of unintended pregnancies. Epidemiol Rev. 2010;32(1):152-74.

7. Rayamajhi RB, Paudel IS, Bhattarai S, Neupane B, Kumar P. Practice of contraception and quality of life among Bhutanese refugee women of Eastern Nepal. Glob J Med Res. 2014;2(2). https://www.medicalresearchjournal.org/ index.php/GJMR/article/view/722. Accessed 16 June 2018.

8. Finer LB, Zolna MR. Declines in unintended pregnancy in the United States, 2008-2011. N Engl J Med. 2016;374(9):843-52.

9. Yazdkhasti M, Pourreza A, Pirak A, Abdi F. Unintended pregnancy and its adverse social and economic consequences on health system: a narrative review article. Iran J Public Health. 2015;44(1):12

10. Habib MA, Raynes-Greenow C, Nausheen S, Soofi SB, Sajid M, Bhutta ZA, et al. Prevalence and determinants of unintended pregnancies amongst women attending antenatal clinics in Pakistan. BMC Pregnancy Childbirth. 2017;17(1):156.

11. Belda SS, Haile MT, Melku AT, Tololu AK. Modern contraceptive utilization and associated factors among married pastoralist women in bale ecoregion, bale zone, south East Ethiopia. BMC Health Serv Res. 2017;17(1):194.

12. Dansereau E, Schaefer A, Hernández B, Nelson J, Palmisano E, Ríos-Zertuche $D$, et al. Perceptions of and barriers to family planning services in the poorest regions of Chiapas, Mexico: a qualitative study of men, women, and adolescents. Reprod Health. 2017;14(1):129.

13. Larivaara MM. Pregnancy prevention, reproductive health risk and morality: a perspective from public-sector women's clinics in St. Petersburg, Russia. Crit Public Health. 2010;20(3):357-71.

14. El-Khoury M, Thornton R, Chatterii M, Kamhawi S, Sloane P, Halassa M. Counseling women and couples on family planning: a randomized study in Jordan. Stud Fam Plan. 2016;47(3):222-38.

15. Najafi-Sharjabad F, Syed Yahya SZ, Rahman HA, Hanafiah M, Abdul MR. Barriers of modern contraceptive practices among asian women: a mini literature review. Global J Health Sci. 2013:5(5):181-92.

16. Ackerson K, Zielinski R. Factors influencing use of family planning in women living in crisis affected areas of sub-Saharan Africa: a review of the literature. Midwifery. 2017;54:35-60.

17. Jordan Communication, Advocacy and Policy Activity (JCAP). Exploring gender norms and family planning in jordan: a qualitative study. 2016. https://jordankmportal.com/resources/exploring-gender-norms-and-familyplanning-in-jordan. Accessed 16 June 2018.

18. Williams SL, Parisi SM, Hess R, Schwarz EB. Associations between recent contraceptive use and quality of life among women. Contraception. 2012; 85(3):282-7

19. Zethraeus N, Dreber A, Ranehill E, Blomberg L, Labrie F, von Schoultz B, et al. A first-choice combined oral contraceptive influences general wellbeing in healthy women: a double-blind, randomized, placebo-controlled trial. Fertil Steril. 2017;107(5):1238-45.

20. Bastianelli C, Farris M, Benagiano G. Use of the levonorgestrel-releasing intrauterine system, quality of life and sexuality. Experience in an Italian family planning center. Contraception. 2011;84(4):402-8.

21. Cristóbal García I, Lete LI, De la Viuda E, Perulero N, Arbart A, Canals I. One year quality of life measured with SEC-QoL in levonorgestrel 52mg IUS users. Contraception. 2016;93(4):367-71

22. Casey PM, MacLaughlin KL, Faubion SS. Impact of contraception on female sexual function. J Women's Health. 2017;26(3):207-13.

23. Hassanin AM, El-Halwagy AM, Ismail NN, Shehab BA. A study of the impact of the commonly used female contraceptive methods in Egypt on female sexual function. J Sex Marital Ther. 2018:44(6):605-12.

24. Umran O. Effect of the contraceptive methods on female sexual function. Int J Caring Sci. 2016;9(3):997-1007.

25. Worly BL, Gur TL, Schaffir J. The relationship between progestin hormonal contraception and depression: a systematic review. Contraception. 2018; 97(6):478-89.

26. Sadatmahalleh SJ, Ziaei S, Kazemnejad A, Mohamadi E. Evaluation of sexual function and quality of life in Iranian women with tubal ligation: a historical cohort study. Int J Impot Res. 2015;27(5):173-7.

27. Ministry of Health. Ministry of health annual statistical book 2018. Amman: Ministry of Health; 2019. http://www.moh.gov.jo/Echobusv3.0/SystemAssets/5 a872ebc-3198-43a0-9f77-e1d7497b3962.pdf. Accessed 12 June 2019

28. Department of Statistics, ICF International. Jordan population and family health survey 2012. Calverton: Department of Statistics and ICF International; 2013. https://hsprogram.com/pubs/pdf/fr282/fr282.pdf. Accessed 28 Oct 2018 
29. Department of Statistics (DOS), ICF. Jordan population and family and health survey 2017-18. Amman, Jordan, and Rockville, Maryland, USA. 2019. https://dhsprogram.com/publications/publication-FR346-DHS-Final-Reports. cfm. Accessed 22 Mar 2019.

30. Bernhart M, Shteiwi M. The contraception-adoption process in Jordan. Prospective study. Amman: CMS Country Research Series; CMS/Jordan, and Jordan Center for Social Research; 2001. https://www.shopsplusproject.org/ sites/default/files/resources/915_file_10_The_Contraception_Adoption_ Process_in_Jordan.pdf. Accessed 22 June 2019

31. Shah R, Kiriya J, Shibanuma A, Jimba M. Use of modern contraceptive methods and its association with QOL among Nepalese female migrants living in Japan. PLoS One. 2018;13(5):e0197243.

32. Bahri N, Tohidinik HR, Bilandi RR, Larki M, Hooshangi F, Soltanian M. The relation between contraception methods and quality of life. Epidemiol Biostat Public Health. 2016;13(4):e11986-1-e11986-7.

33. Pérez-Campos $E$, Dueñas $J$, de la Viuda $E$, Gómez MÁ, Lertxundi $R$, Sánchez-Borrego R, et al. Development and validation of the SEC-QoL questionnaire in women using contraceptive methods. Value Health. 2011;14(6):892-9.

34. Li RH, Lo SS, Teh DK, Tong N-C, Tsui MH, Cheung K-B, et al. Impact of common contraceptive methods on quality of life and sexual function in Hong Kong Chinese women. Contraception. 2004;70(6):474-82.

35. Kar N, Swain SP, Patra S, Kar B. The WHOQOL-BREF: translation and validation of the odia version in a sample of patients with mental illness. Indian J Soc Psychiatry. 2017;33(3):269-73.

36. Nedjat S, Montazeri A, Holakouie K, Mohammad K, Majdzadeh R. Psychometric properties of the Iranian interview-administered version of the World Health Organization's Quality of Life Questionnaire (WHOQOL-BREF): a population-based study. BMC Health Serv Res. 2008;8:61 https://doi.org/10. 1186/1472-6963-8-61.

37. Golicki D, Styczen P, Szczepkowski M. Quality of life in stoma patients in Poland: multicentre cross-sectional study using WHOQOL-BREF questionnaire. Przegl Epidemiol. 2013;67(3):491-6.

38. World Health Organization. Programme on mental health: WHOQOL user manual. Geneva: World Health Organization. http://apps.who.int/iris/ handle/10665/77932. Accessed 11 May 2018.

39. World Health Organization. WHOQOL-BREF: introduction, administration, scoring and generic version of the assessment: field trial version, December 1996. Geneva: World Health Organization; 1996. http://www.who.int/ mental_health/media/en/76.pdf. Accessed 11 May 2018

40. Dawass M. Poverty in Jordan. Amman: Department of Statistics DOS; 2015. http://www.unece.org/fileadmin/DAM/stats/documents/ece/ces/ge.15/2015/ WP_11_EN_Jordan.pdf. Accessed 17 May 2018

41. Ajluni S, Kawar M. The impact of the Syrian refugee crisis on the labour market in Jordan: A preliminary analysis. International labour organization: regional office for the arab states. 2014. https://www.lo.org/beirut/ publications/WCMS_242021/lang--en/index.htm. Accessed 17 May 2018.

42. Abdulrahim S, Jawad M. Socioeconomic differences in smoking in Jordan, Lebanon, Syria, and Palestine: a cross-sectional analysis of national surveys. PLoS One. 2018;13(1):e0189829.

43. Department of Statistics. Labor Statistics in Jordan 2012-2016. In: Department of Statistics, editor. 2018. http://dosweb.dos.gov.jo/DataBank/ JOBS/Jobs2012-2016.pdf. Accessed 124 Oct 2018.

44. Toffol E, Heikinheimo O, Koponen P, Luoto R, Partonen T. Hormonal contraception and mental health: results of a population-based study. Hum Reprod. 2011;26(11):3085-93.

45. Bongaarts J, Cleland J, Townsend JW, Bertrand JT, Gupta MD. Family planning programs for the 21 st century. New York: Population Council; 2012. https://www.popcouncil.org/uploads/pdfs/2012_FPfor21stCentury.pdf. Accessed 11 July 2018

46. United States Agency for International Development (USAID) and Jordan Communication, Advocacy and Policy Activity. Family planning among Syrian refugees in Jordan. Amman: Jordan Communication, Advocacy, and Policy Activity. https://jordankmportal.com/resources/family-planningamong-syrian-refugees-in-jordan. Accessed 11 July 2018

47. Sudanagunta S. Association of basic maternal factors and maternal autonomy with malnourishment among children 6-36 months old in an urban slum in Bangalore, India. Dissertation Dallas: The University of Texas Southwestern Medical Center; 2016.

48. Ghule M, Raj A, Palaye P, Dasgupta A, Nair S, Saggurti N, et al. Barriers to use contraceptive methods among rural young married couples in
Maharashtra, India: qualitative findings. Asian J Res Soc Sci Humanit. 2015; 5(6):18-33.

49. Naz B, Batool SS. Infertility related issues and challenges: perspectives of patients, spouses, and infertility experts. Pak J Soc Clin Psychol. 2017; 15(2):3-11.

50. Spencer D. To protect her honour. Child marriage in emergencies-the fatal confusion between protecting girls and sexual violence. UK: CARE International UK; 2015. https://data2.unhcr.org/en/documents/download/5 9751. Accessed 11 July 2018

51. Chandra-Mouli V, McCarraher DR, Phillips SJ, Williamson NE, Hainsworth G. Contraception for adolescents in low and middle income countries: needs, barriers, and access. Reprod Health. 2014;11(1):1.

52. Yadav B, Batra A, Egbert M. Reproductive health issues amongst adolescent females attending a health centre in Rural Haryana: a wake up call. J Evol Med Dent Sci. 2015;4(90):15502-7. https://doi.org/10. 14260/jemds/2015/2218.

53. Institute for Reproductive Health. Final Report: Jordan family planning assessment. FACT project. Washington, D.C: Institute for Reproductive Health, Georgetown University; 2016. http://irh.org/wp-content/ uploads/2016/05/USAID_FP_assessment_2016_IRH_FINAL_REPORT.pdf. Accessed 29 June 2019

54. Maffi I. Women, health and the state in the middle east. the politics and culture of childbrth in jordan. Library of Modern Middle East Studies (Book 113). London: I.B. Tauris; 2012.

55. Singh K, Bharati G, Prasad D, Kumari S. Role of levonorgestrel releasing intrauterine device in management of heavy menstrual bleeding: a conservative approach. Int J Reprod Contracept Obstet Gynecol. 2017; 6(2):631-5.

56. Rowe P, Farley T, Peregoudov A, Piaggio G, Boccard S, Landoulsi S, et al. Safety and efficacy in parous women of a 52-mg levonorgestrel-medicated intrauterine device: a 7-year randomized comparative study with the TCu380A. Contraception. 2016;93(6):498-506.

57. Polis $C B$, Hussain $R$, Berry $A$. There might be blood: a scoping review on women's responses to contraceptive-induced menstrual bleeding changes. Reprod Health. 2018;15(1):114 https://doi.org/10.1186/s12978-018-0561-0.

58. Nelson AL. Male condoms. In: Shoupe D, editor. Contraception. Gynecology in practice. Oxford: UK: Wiley-Blackwell; 2011. p. 114-22.

59. Tadayon $M$, Honarjoo $M$, Abbaspoor Z. The relationship of male condoms and withdrawal contraceptive methods with female sexual function and satisfaction: a cross sectional study. Int J Reprod Contracept Obstet Gynecol. 2017;5(8):2630 https://doi.org/10.18203/2320-1770.jircog20162634.

60. Matsumoto Y, Yamabe S, Ideta K. Quality of life among Japanese oral contraceptive users. In: Preedy VR, Watson RR, editors. Handbook of disease burdens and quality of life measures. New York: Springer; 2010. p. 1937-50.

61. Goodarzi F, Hossieni M, Moomeni E. Impact of oral contraceptives on the quality of life of women referred to health centers of Yasuj, Iran. Armaghane danesh. 2014;19(3):242-51.

62. Zhao J, Li Y, Wu Y, Zhou J, Ba L, Gu X, et al. Impact of different contraceptive methods on quality of life in rural women of the Jiangsu province in China. Contraception. 2009;80(2):180-6.

63. Di Carlo C, Sansone A, De Rosa N, Gargano V, Tommaselli GA, Nappi C, et al. Impact of an implantable steroid contraceptive (etonogestrel-releasing implant) on quality of life and sexual function: a preliminary study. Gynecol Endocrinol. 2014;30(1):53-6.

64. Amico J, Kumar B, Rosenstein H, Gold M. The contraceptive implant: an updated review of the evidence. Curr Obstet Gynecol Rep. 2015;4(1):79-88.

65. Wanyonyi SZ, Stones WR, Sequeira E. Health-related quality of life changes among users of depot medroxyprogesterone acetate for contraception. Contraception. 2011;84(5):e17-22 https://doi.org/10.1016/j.contraception. 2011.05.022

66. Lutala PM, Hugo JF, Luhiriri LN. Psychosocial implications of tubal ligation in a rural health district: A phenomenological study. Reprod Health. 2011;8(1): 38 http://www.reproductive-health-journal.com/content/8/1/38.

67. Bahrami N, Karimian Z, Bahrami S, Raji M, Nosratabadi M. Comparison of quality of life of women on different methods of contraception. Jundishapur Sci Med J. 2016;15(4):443-542.

68. Akpor O, Fadare R, Ei E. Knowledge and perception of women regarding bilateral tubal ligation in Southwest Nigeria. IOSR J Nurs Health Sci. 2016;05:31-6.

69. Firouzabadi A, Noori A, Naseri H. Evaluation of depression after tubal ligation. J Reprod Infertil. 2002;3(3):52-8. 
70. Gulum M, Yeni E, Sahin M, Savas M, Ciftci H. Sexual functions and quality of life in women with tubal sterilization. Int J Impot Res. 2010;22(4):267-71.

71. Parsanezhad M, Alborzi S, Jahromi BN. Menstrual abnormalities and pain after five tubal sterilization methods: a randomized controlled trial. Iran J Med Sci. 2015;28(2):51-6.

72. Sadatmahalleh SJ, Ziaei S, Kazemnejad A, Mohamadi E. Menstrual pattern following tubal ligation: a historical cohort study. Int J Fertil Steril. 2016;9(4):477-82.

73. Howard B, Grubb E, Lage MJ, Tang B. Trends in use of and complications from intrauterine contraceptive devices and tubal ligation or occlusion. Reprod Health. 2017;14(1):70 https://doi.org/10.1186/s12978-017-0334-1.

74. Villavicencio J, Allen RH. Unscheduled bleeding and contraceptive choice: increasing satisfaction and continuation rates. Open access journal of contraception. Open Access J Contracept. 2016;7:43-52 https://doi.org/10. 2147/OAJC.S85565.

75. Yoost J. Understanding benefits and addressing misperceptions and barriers to intrauterine device access among populations in the United States. Patient Prefer Adherence. 2014;8:947-57 https://doi.org/10.2147/PPA.S45710

76. Peipert JF, Zhao Q, Allsworth JE, Petrosky E, Madden T, Eisenberg D, et al. Continuation and satisfaction of reversible contraception. Obstet Gynecol. 2011;117(5):1105-13.

77. Choi Y. Estimates of side effects counseling in family planning using three data sources: implications for monitoring and survey design. Stud Fam Plan. 2018:49(1):23-39.

78. Assaf S, Wang W, Mallick L. Provider counseling and knowledge transfer in health facilities of Haiti Malawi and Senegal. DHS analytical studies no. 60 Rockville: ICF International; 2016. https://dhsprogram.com/pubs/pdf/AS60/ AS60.pdf. Accessed 22 June 2019

79. Belbeisi A, Zindah M, Walke H, Jarrar B, Mokdad A. Health related quality of life measures by demographics and common health risks, Jordan 2004. Int J Public Health. 2009;54(Suppl 1):106 https://doi.org/10. 1007/s00038-009-0014-1.

80. Rominski SD, Sk Morhe E, Maya E, Manu A, Dalton VK. Comparing women's contraceptive preferences with their choices in 5 urban family planning clinics in Ghana. Glob Health Sci Pract. 2017;5(1):65-74 https://doi.org/10. 9745/GHSP-D-16-00281.

81. Titaley CR, Wijayanti RU, Damayanti R, Setiawan AD, Dadun DD, et al. Increasing the uptake of long-acting and permanent methods of family planning: A qualitative study with village midwives in East Java and Nusa Tenggara Barat provinces, Indonesia. Midwifery. 2017;53:55-62.

82. Gbagbo FY, Kayi EA. Use and discontinuation of intrauterine contraceptive device in the Greater Accra region of Ghana. Contracept Reprod Med. 2018; 3(1):8 https://doi.org/10.1186/s40834-018-0061-1.

83. Alyahya MS, Khader YS, Batieha A, Asad M. The quality of maternal-fetal and newborn care services in Jordan: a qualitative focus group study. BMC Health Serv Res. 2019;19(1):425 https://doi.org/10.1186/s12913-019-4232-9.

84. Ertekin Pinar S, Demirel G, Yildirim G, Daglar G. Sexual experiences and quality of life in Turkish women using methods of contraception. J Obstet Gynaecol. 2019;39(6):782-7 https://doi.org/10.1080/01443615.2019.1581738

85. Wulifan J, Bagah DA. Male involvement in family planning in muslim communities in Wa municipality, Ghana. J Humanit Soc Sci. 2015;5(7):86-97.

86. Kriel Y, Milford C, Cordero J, Suleman F, Beksinska M, Steyn P, Smit JA. Male partner influence on family planning and contraceptive use: perspectives from community members and healthcare providers in KwaZulu-Natal. S Afr Reprod Health. 2019;16:89 https://doi.org/10.1186/s12978-019-0749-y.

87. Sharma S, Bhuvan KC, Khatri A. Factors influencing male participation in reproductive health: a qualitative study. J Multidiscip Healthc. 2018;11:601 https://doi.org/10.2147/JMDH.S176267.

88. Kohan S, Simbar M, Taleghani F. Empowerment in family planning as viewed by Iranian women: A qualitative study. J Biosoc Sci. 2012; 44(2):209-19.

89. Eqtait FA, Abushaikha L. Male involvement in family planning: an integrative review. Open J Nurs. 2019;9(3):294-302.

\section{Publisher's Note}

Springer Nature remains neutral with regard to jurisdictional claims in published maps and institutional affiliations.

\section{Ready to submit your research? Choose BMC and benefit from:}

- fast, convenient online submission

- thorough peer review by experienced researchers in your field

- rapid publication on acceptance

- support for research data, including large and complex data types

- gold Open Access which fosters wider collaboration and increased citations

- maximum visibility for your research: over $100 \mathrm{M}$ website views per year

At BMC, research is always in progress.

Learn more biomedcentral.com/submissions 